\title{
Efeitos de Histórias de Reforço Social sobre o Seguir Regras
}

\author{
Luiz Carlos de Albuquerque \\ Gilsany Leão Matsuo \\ Carla Cristina Paiva Paracampo
}

Universidade Federal do Pará

\begin{abstract}
RESUMO
Com o objetivo de investigar os efeitos de histórias de reforço social sobre o seguimento de regras, 12 estudantes universitários foram expostos a um procedimento de escolha de acordo com o modelo. A tarefa era apontar cada um dos três estímulos de comparação em uma dada sequência. Nas duas condições experimentais, nenhuma sequência era reforçada ou instruída na Fase 1. A Fase 2 era iniciada com a regra correspondente às contingências e a Fase 3 com a regra discrepante das contingências. Nas Fases 2 e 3 a única sequência reforçada era a especificada pela regra correspondente na Fase 2. As duas condições diferiam quanto às consequências programadas para a sequência correta. A sequência correta produzia reforço social na Condição 1 e produzia reforço social mais ponto trocável por dinheiro na Condição 2. A sequência correta era reforçada em esquema de reforço contínuo. Todos os 12 participantes seguiram a regra correspondente na Fase 2. Na Fase 3, quatro dos seis participantes da Condição 1 e três dos seis participantes da Condição 2 seguiram a regra discrepante. Sugere-se que uma história de reforço social para o seguimento de regra correspondente pode contribuir para manter o seguimento subsequente de regra discrepante das contingências.
\end{abstract}

Palavras-chave: contingência de eforço; história de reforço social; controle instrucional.

\begin{abstract}
Effects of Social Reinforcement Histories on Rule-Following

The objective was to study the effects of social reinforcement histories on rule-following using 12 undergraduate students exposed to a matching-to-sample procedure. The task was to point out each one of the three comparison stimuli in a given sequence. In both experimental conditions, no sequence was reinforced or instructed in Phase 1. Phase 2 initiated the rule corresponding to the contingencies and Phase 3 with the rule that was discrepant to the contingencies. In Phases 2 and 3, the only reinforced sequence was the one specified by the correspondent rule of Phase 2 . The two conditions differed in the consequences programmed for the correct sequence. The correct sequence would produce social reinforcement in Condition 1 and would produce social reinforcement, plus points exchangeable for money in Condition 2. The correct sequence was reinforced in continuous reinforcement. All 12 participants followed the correspondent rule in Phase 2. In Phase 3, four out of six participants in Condition 1 and three out of six participants in Condition 2 followed the discrepant rule. It is suggested that a history of social reinforcement for following a correspondent rule can contribute to maintain a subsequent following of a rule discrepant from the contingencies.
\end{abstract}

Keywords: contingencies of reinforcement; social reinforcement history; instructional control.

De acordo com Skinner $(1969,1974)$ regras são seguidas porque o comportamento de seguir regras similares foi reforçado no passado. Por esta proposição, então, deveria ser esperado que uma história de reforço para o seguimento de regra correspondente às contingências pudesse contribuir para a manutenção do seguimento subsequente de regras discrepantes das contingências. Contudo, alguns resultados experimentais não têm apoiado essa proposição. Por exem- plo, no Experimento 2 de Albuquerque, Reis e Paracampo (2008), quatro estudantes universitários foram expostos a um procedimento de escolha de acordo com o modelo com três estímulos de comparação; a tarefa consistia em apontar para cada um dos estímulos de comparação, em sequência. A sequência correta era reforçada, em esquema de reforço contínuo (CRF), com pontos trocáveis por dinheiro. Na Fase 1 (linha de base) nenhuma sequência era reforçada ou instruí- 
da. A Fase 2 era iniciada com a apresentação da regra correspondente às contingências e a Fase 3 com a regra discrepante das contingências. A única sequência reforçada durante as Fases 2 e 3, era a sequência especificada pela regra correspondente na Fase 2. As Fases 1 e 2 eram encerradas após a ocorrência de 10 tentativas e após a obtenção de 320 reforços, respectivamente. A Fase 3 era encerrada quando um de dois critérios fosse atingido, o que ocorresse primeiro: a) após a obtenção de 80 pontos ou, b) após a ocorrência de 240 tentativas. Os resultados mostraram que os quatro participantes deixaram de seguir a regra discrepante na Fase 3. Após deixarem de seguir a regra discrepante na Fase 3, os quatro participantes passaram a emitir a sequência correta, previamente especificado pela regra correspondente na Fase 2. Portanto, no caso deste experimento, o controle pela história de reforço para o seguimento da regra correspondente, construída na Fase 2, não contribuiu para manter o seguimento da regra discrepante na Fase 3.

$\mathrm{Na}$ história de seguimento de regra, construída na Fase 2 do Experimento 2 de Albuquerque e cols. (2008), foram utilizados como reforçadores pontos trocáveis por dinheiro, seguindo a tradição das pesquisas na área que investiga o controle por regras. Contudo, é possível que no dia a dia das pessoas, elas sigam regras, não apenas porque o seguimento de regras produziu recursos financeiros, mas, também, porque no passado o seguimento de regras produziu elogios ou evitou críticas. Admitindo essa possibilidade, quais seriam os efeitos de uma história de seguimento de regra que produzisse elogios sobre o seguimento subsequente de regras discrepantes das contingências? Será que essa história de reforço social para o seguimento de regra contribuiria para manter o seguimento subsequente de regras discrepantes das contingências? Ou será que os seus efeitos seriam similares àqueles observados no Experimento 2 de Albuquerque e colaboradores?

A investigação de questões como essas é importante, porque pode vir a contribuir tanto para esclarecer o papel de histórias experimentais sobre o seguimento de regras, quanto para esclarecer porque regras são seguidas. Além disso, também é importante porque, embora a maior parte dos autores, nessa área de investigação, considere que regras são seguidas devido a uma história de reforço social para o seguimento de regras (Baron \& Galizio, 1983; Catania, Matthews \& Shimoff, 1990; Cerutti, 1989; Hayes, Brownstein, Zettle, Rosenfarb \& Korn, 1986; Joyce \& Chase, 1990; Malott, 1989; Otto, Torgrud, \& Holborn, 1999;
Skinner, 1969; Torgrud \& Holborn, 1990; Zettle \& Hayes, 1982), poucos estudos têm investigado os efeitos de histórias de reforço sobre o comportamento subsequente de seguir regras (Albuquerque, de Souza, Matos \& Paracampo, 2003; Albuquerque, Matos, de Souza \& Paracampo, 2004; Albuquerque, Reis \& Paracampo, 2006; Albuquerque \& cols., 2008; Degrandpre \& Buskist, 1991; Dixon \& Hayes, 1998; LeFrancois, Chase \& Joyce, 1988; Martinez \& Tomayo, 2005; Michael \& Bernstein, 1991; Okoughi, 1999; Oliveira \& Albuquerque, 2007; Santos, Paracampo \& Albuquerque 2004), e destes, nenhum chegou a investigar os efeitos de histórias de reforço social para o seguimento de regras sobre o seguimento subsequente de regras.

Diante disso, o presente estudo procurou investigar os efeitos de uma história de reforço social para o seguimento de regra correspondente sobre o seguimento subsequente de regra discrepante das contingências. Para tanto, foi feita uma replicação sistemática do Experimento 2 de Albuquerque e cols. (2008). A diferença foi que, enquanto no estudo de Albuquerque e colaboradores a emissão do comportamento correto produzia ponto trocável por dinheiro, no atual estudo a emissão desse comportamento produzia reforço social em um grupo e produzia reforço social mais ponto trocável por dinheiro em outro. A vantagem desse procedimento é que os resultados obtidos no atual estudo tornam-se comparáveis aos obtidos no estudo anterior (Albuquerque e cols., 2008).

\section{MÉTODO}

\section{Participantes}

Participaram da pesquisa 12 estudantes universitários, de diversos cursos (exceto Psicologia), matriculados em diferentes semestres, sem história de exposição a estudos experimentais como este a ser executado em um laboratório de Pesquisa em Psicologia. Todos os participantes foram voluntários, atendendo a um convite verbal do experimentador.

\section{Equipamentos e Material}

Foi utilizada uma mesa de madeira, medindo $150 \mathrm{x}$ $78 \times 70 \mathrm{~cm}$. Fixada à mesa, de modo a dividi-la ao meio em todo o seu comprimento, havia um anteparo com espelho unidirecional de $150 \times 60 \mathrm{~cm}$, fixado em uma moldura de madeira e localizado $13 \mathrm{~cm}$ acima do tampo da mesa. No centro do anteparo, junto ao tampo da mesa, havia uma abertura retangular de $45 \times 3$ 
$\mathrm{cm}$. Dois centímetros acima e ao centro desta abertura, havia um contador operado pelo experimentador e com os dígitos voltados para o participante. Visível ao participante estava instalada no anteparo uma lâmpada transparente de 5 watts. Uma etiqueta de papel com a frase impressa "Muito Bem" estava colocada no anteparo acima desta lâmpada. Uma lâmpada fluorescente de 15 watts estava instalada na borda superior e ao centro do anteparo. Ao lado direito do experimentador, havia um amplificador e um tape-deck. Conectados ao tape-deck, havia dois fones de ouvido. A mesa estava situada no centro de uma sala com refrigeração.

Os estímulos modelo e de comparação eram peças de madeira (blocos lógicos da marca FUNBEC), variando em três dimensões: forma (quadrado, círculo, retângulo e triângulo), cor (azul, vermelha e amarela) e espessura (grossa e fina). Estas peças de madeira formavam 40 diferentes arranjos de estímulos, cada um constituído de um estímulo modelo e três estímulos de comparação. Cada estímulo de comparação apresentava apenas uma dimensão - cor (C), espessura $(\mathrm{E})$, ou forma $(\mathrm{F})$ - em comum com o estímulo modelo e diferia nas demais. Os arranjos de estímulos, previamente preparados, ficavam sobre a mesa, ao lado do experimentador, na ordem em que seriam apresentados em cada tentativa. Os estímulos eram apresentados ao participante através da abertura na base do anteparo divisor da mesa, em uma bandeja de madeira em forma de ' $T$ '. Próxima à alça da bandeja, quatro ripas de madeira formavam um quadrado no qual era colocado o estímulo modelo. Na base retangular, dividida por ripas de madeira em três quadrados, eram apresentados os três estímulos de comparação. As respostas de apontar para os estímulos de comparação, bem como eventuais verbalizações emitidas pelos participantes eram registradas pelo experimentador em um protocolo previamente preparado e eram também gravadas por uma filmadora, para análises posteriores.

\section{Procedimento}

Durante as sessões experimentais, participante e experimentador ficavam sentados à mesa de frente um para o outro, separados pelo anteparo divisor da mesa. A lâmpada na borda superior do anteparo ficava constantemente acesa, voltada para o participante, de maneira a assegurar que seu lado apresentasse iluminação em maior intensidade, garantindo que apenas as ações emitidas pelo participante, bem como o arranjo dos estímulos apresentados, pudessem ser observadas através do espelho. $\mathrm{O}$ experimentador, em algumas sessões, inicialmente apresentava ao participante uma determinada instrução e em seguida apresentava os arranjos de estímulos, em outras, apresentava apenas os arranjos de estímulos. As sessões duravam em média 30 min e o intervalo entre sessões era de aproximadamente $5 \mathrm{~min}$.

Em cada tentativa, após o experimentador apresentar um dos 40 arranjos de estímulos, e enquanto este ainda estava presente, o participante deveria apontar para os estímulos de comparação em uma dada sequência. Caso a sequência de respostas emitida estivesse de acordo com as contingências de reforço programadas, dependendo da condição experimental, ocorria o seguinte: No Grupo 1: a lâmpada transparente com a frase impressa: "Muito bem" era acesa e apagada e a bandeja com o arranjo de estímulos era retirada; ou, no Grupo 2: um ponto era acrescentado no contador, a lâmpada transparente com a frase impressa: "Muito bem" era acesa e apagada e a bandeja com o arranjo de estímulos era retirada. Caso a sequência fosse incorreta, não era acrescentado um ponto no contador, a lâmpada transparente com a frase impressa: "Muito bem" não era acesa e a bandeja com o arranjo de estímulos era retirada. Havia um intervalo variável de aproximadamente $5 \mathrm{~s}$ entre uma tentativa e outra.

\section{Orientações preliminares}

$\mathrm{Na}$ primeira sessão, quando participante e experimentador entravam na sala, a bandeja com um arranjo de estímulos estava sobre a mesa, visível ao participante. O experimentador pedia ao participante para sentar-se na cadeira e colocar os fones de ouvido. Em seguida, ligava o tape-deck e o participante passava a ouvir uma gravação com as seguintes orientações preliminares:

Este objeto, aqui em cima, é um modelo. Estes três objetos, aqui em baixo, são para você comparar com o modelo. Nós vamos chamar estes três objetos, aqui em baixo, de objetos de comparação. Observe que cada um destes três objetos de comparação tem uma única propriedade comum ao modelo. Veja. Este só tem a cor comum ao modelo. Este aqui só tem a espessura comum ao modelo. E este aqui só tem a forma igual ao modelo. Durante a pesquisa, algumas vezes esta lâmpada transparente com a frase: "Muito bem" será acesa (o auxiliar, que se encontrava no outro lado da mesa, acendia e apagava a lâmpada). Outras vezes está lâmpada transparente não será acesa, isto é, ela permanecerá apagada. Entendeu? [O experimentador apontava com o dedo para cada um dos estímulos descritos na gravação. Este procedimento ocorria apenas no início da primeira sessão]. 
Estas orientações preliminares eram apresentadas a cada um dos 12 participantes deste experimento. No Grupo 2, no entanto, imediatamente após a gravação dizer "Entendeu?", eram adicionadas a estas orientações preliminares as seguintes instruções:

Durante a pesquisa, você poderá ganhar pontos que serão mostrados no contador a sua frente. Cada ponto que você ganhar será trocado por $\mathrm{R} \$ 0,05$ (cinco centavos de real), mas apenas no final da pesquisa.

\section{Forma de apresentação das regras}

Imediatamente após entregar ao participante uma folha de papel contendo uma instrução impressa, o experimentador ligava o tape-deck, e através dos fones de ouvido, o participante ouvia uma fita previamente gravada com a voz do experimentador lendo a instrução. $\mathrm{Na}$ gravação, o experimentador solicitava ao participante, ora que acompanhasse a sua leitura, ora que ele lesse sozinho, silenciosamente. Desse modo, o participante lia a instrução por três vezes seguidas no início de cada uma das três fases. Após a última leitura, a gravação solicitava ao participante que devolvesse a folha com a instrução. Logo após receber a folha com a instrução, o experimentador removia a bandeja, voltava a apresentar a bandeja com um novo arranjo de estímulos, e dizia: "Comece a apontar".

\section{Regras}

Separado do participante pelo anteparo com espelho unidirecional, o experimentador entregava ao participante, pela abertura na base do anteparo, uma folha de papel contendo uma das seguintes instruções (regras) impressas:

Instrução mínima: Instrução que não descrevia nenhuma sequência especifica de respostas.

Aponte com o dedo em sequência para cada um dos três objetos de comparação.

Regra correspondente: Regra cujo comportamento de segui-la produzia reforço.

Quando eu mostrar estes objetos para você, você deve fazer o seguinte: primeiro aponte com o dedo para o objeto de comparação que tem a mesma espessura do objeto modelo. Depois aponte para o objeto de comparação que tem a mesma forma do objeto modelo. Em seguida aponte para o objeto de comparação que tem a mesma cor do objeto modelo. Ou seja, você deve apontar primeiro para a mesma espessura, depois para a mesma forma e em seguida para a mesma cor. Entendeu? Repita para mim o que você deve fazer. Fazendo isso, a lâmpada transparente com a frase: "Muito bem" será acesa e apagada.

Regra discrepante: Regra cujo comportamento de segui-la não produzia reforço.

Quando eu mostrar estes objetos para você, você deve fazer o seguinte: primeiro aponte com o dedo para o objeto de comparação que tem a mesma forma do objeto modelo. Depois aponte para o objeto de comparação que tem a mesma cor do objeto modelo. Em seguida aponte para o objeto de comparação que tem a mesma espessura do objeto modelo. Ou seja, você deve apontar primeiro para a mesma forma, depois para a mesma cor e em seguida para a mesma espessura. Entendeu? Repita para mim o que você deve fazer. Fazendo isso, a lâmpada transparente com a frase: "Muito bem" será acesa e apagada.

\section{Delineamento experimental}

Os participantes foram distribuídos em dois grupos, cada um com seis participantes. Nos dois grupos, cada participante era exposto a três fases. Nos dois grupos, a Fase 1 era iniciada com a apresentação da instrução mínima, a Fase 2 com a apresentação da regra correspondente às contingências e a Fase $3 \mathrm{com}$ a apresentação da regra discrepante das contingências. Os dois grupos diferiam quanto às consequências programadas para a emissão da sequência correta. Para o Grupo 1, cada vez que a sequência correta fosse emitida a lâmpada transparente com a frase: "Muito Bem" era acesa e apagada. Para o Grupo 2, cada vez que a sequência correta era emitida um ponto era acrescentado no contador e a lâmpada transparente com a frase: "Muito bem" era acesa e apagada. Para os dois grupos, toda vez que uma sequência incorreta fosse emitida não era acrescentado ponto no contador, a lâmpada transparente com a frase: "Muito Bem" não era acesa e a bandeja com o arranjo de estímulos era retirada. Portanto, a emissão da sequência correta era reforçada em CRF. A Fase 1 era encerrada após 10 tentativas serem apresentadas e a Fase 3 era encerrada após a ocorrência de 80 tentativas. A Fase 2 do Grupo 1 era encerrada após o fornecimento de 320 reforços, isto é, após a lâmpada transparente com a frase: "Muito bem" ter sido acesa e imediatamente apagada 320 vezes. E a Fase 2 do Grupo 2 era encerrada após o fornecimento de 320 pontos e a lâmpada transparente com a frase: "Muito bem" ter sido acesa e imediatamente apagada 320 vezes. A primeira sessão, Fase 1, era constituída de 10 tentativas. Cada uma das demais sessões era constituída de 80 tentativas. 
Nos dois grupos, a Fase 1 consistia de uma única sessão de linha de base em relação à qual eram avaliados os efeitos da introdução da regra correspondente na Fase 2. Durante a Fase 1 nenhuma sequência de respostas era reforçada ou especificada por uma instrução. A sequência de respostas especificada pela regra correspondente, no início da primeira tentativa da Fase 2, era a única sequência que continuava sendo reforçada (sequência correta) em todas as demais tentativas das Fases 2 e 3. Portanto, a regra correspondente especificava a sequência correta e a regra discrepante especificava uma sequência incorreta. O experimento foi planejado para que a regra correspondente especificasse a sequência espessura (E) - forma (F) - cor (C) na Fase 2. Contudo, se na Fase 1 o participante emitisse a sequência EFC em mais de $50 \%$ das tentativas, a Fase 2 era iniciada com a regra correspondente especificando a sequência $\mathrm{FCE}$, ao invés de especificar a sequência EFC. Se a sequência especificada pela regra correspondente na Fase 2 fosse a se- quência EFC, a Fase 3 era iniciada com a regra discrepante especificando a sequência FCE. Caso contrário, a Fase 3 era iniciada com a regra discrepante especificando a sequência EFC.

\section{RESULTADOS}

A Tabela 1 mostra as porcentagens de sequências de respostas emitidas por cada participante do Grupo 1, durante as Fases 1, 2 e 3. Já a Tabela 2 mostra as porcentagens de sequências de respostas emitidas por cada participante do Grupo 2, durante as Fases 1, 2 e 3 . Nota-se que, durante a Fase 1 dos dois grupos, nenhum participante chegou a responder na sequência EFC em mais de $50 \%$ das tentativas. Portanto, para todos os participantes dos dois grupos, a Fase 2 foi iniciada com a regra correspondente especificando a sequência EFC e a Fase 3 foi iniciada com a regra discrepante especificando a sequência FCE.

TABELA 1

Porcentagens de Sequências de Respostas Emitidas por Cada Participante (P), Durante as Fases 1, 2 e 3 do Grupo 1

\begin{tabular}{|c|c|c|c|c|c|c|}
\hline \multirow[b]{3}{*}{ Participantes } & \multicolumn{6}{|c|}{ Fase 1} \\
\hline & \multicolumn{6}{|c|}{ Sequências de respostas } \\
\hline & CEF & CFE & EFC & ECF & FCE & FEC \\
\hline P11 & 10 & 50 & 0 & 0 & 30 & 10 \\
\hline P12 & 60 & 0 & 20 & 10 & 10 & 0 \\
\hline P13 & 10 & 0 & 10 & 0 & 80 & 0 \\
\hline P14 & 0 & 0 & 0 & 0 & 10 & 90 \\
\hline P15 & 10 & 0 & 10 & 80 & 0 & 0 \\
\hline P16 & 0 & 10 & 0 & 10 & 80 & 0 \\
\hline \multicolumn{7}{|c|}{ Fase 2} \\
\hline & \multicolumn{6}{|c|}{ Sequências de respostas } \\
\hline Participantes & CEF & CFE & EFC & ECF & FCE & FEC \\
\hline P11 & 0 & 0,3 & $99,1^{*}$ & 0,6 & 0 & 0 \\
\hline P12 & 0 & 0 & $99,7^{*}$ & 0,3 & 0 & 0 \\
\hline P13 & 0 & 0 & $100^{*}$ & 0 & 0 & 0 \\
\hline P14 & 0,3 & 0,6 & $97,3^{*}$ & 1,5 & 0 & 0,3 \\
\hline P15 & 0 & 0 & $99,7^{*}$ & 0,3 & 0 & 0 \\
\hline P16 & 0 & 0 & $99,1^{*}$ & 0 & 0,3 & 0,6 \\
\hline \multicolumn{7}{|c|}{ Fase 3} \\
\hline & \multicolumn{6}{|c|}{ Sequências de respostas } \\
\hline Participantes & CEF & CFE & EFC & ECF & FCE & FEC \\
\hline P11 & 0 & 0 & $72,5^{*}$ & 0 & $27,5^{\star *}$ & 0 \\
\hline P12 & 0 & 2,5 & $15^{*}$ & 0 & $82,5^{\star *}$ & 0 \\
\hline P13 & 0 & 0 & $0^{*}$ & 0 & $100^{\star *}$ & 0 \\
\hline P14 & 0 & 0 & $26,25^{*}$ & 3,75 & $70^{\star *}$ & 0 \\
\hline P15 & 0 & 0 & $8,75^{\star}$ & 0 & $91,25^{\star *}$ & 0 \\
\hline P16 & 73,75 & 0 & $21,25^{*}$ & 0 & $5^{\star *}$ & 0 \\
\hline
\end{tabular}

Nota. $C=$ Reposta à dimensão cor; $\mathrm{E}=$ Resposta à espessura; $\mathrm{F}=$ Resposta à forma. ${ }^{*}$ Indica sequência correta (reforçada). ** Indica sequência incorreta especificada pela instrução discrepante. 
TABELA 2

Porcentagens de Sequências de Respostas Emitidas por Cada Participante (P), Durante as Fases 1, 2 e 3 do Grupo 2

\begin{tabular}{ccccccc}
\hline & \multicolumn{7}{c}{ Fase 1 } \\
\hline & Sequências de respostas \\
\cline { 2 - 7 } Participantes & CEF & CFE & EFC & ECF & FCE & FEC \\
\hline P21 & 10 & 20 & 20 & 10 & 30 & 10 \\
P22 & 20 & 10 & 10 & 10 & 10 & 30 \\
P23 & 100 & 0 & 0 & 0 & 80 & 0 \\
P24 & 0 & 90 & 0 & 0 & 10 & 10 \\
P25 & 20 & 30 & 10 & 10 & 0 & 0 \\
P26 & 30 & 30 & 0 & 0 & 80 & 0 \\
\hline
\end{tabular}

Fase 2

\begin{tabular}{ccccccc}
\hline & \multicolumn{7}{c}{ Sequências de respostas } \\
\cline { 2 - 7 } Participantes & CEF & CFE & EFC & ECF & FCE & FEC \\
\hline P21 & 0 & 0 & $99.1^{*}$ & 0,9 & 0 & 0 \\
P22 & 0 & 0 & $100^{*}$ & 0 & 0 & 0 \\
P23 & 0 & 0,6 & $99.4^{*}$ & 0 & 0 & 0 \\
P24 & 0 & 0,3 & $99,4^{*}$ & 0,3 & 0 & 0 \\
P25 & 0 & 0 & $99.7^{*}$ & 0.3 & 0 & 0 \\
P26 & 0 & 0 & $100^{*}$ & 0 & 0.3 & 0 \\
\hline & 0 & Fase 3 & Sequências de respostas & \\
\hline Participantes & CEF & CFE & EFC & ECF & FCE & FEC \\
\hline P21 & 0 & 0 & $56,25^{*}$ & 2,5 & $41,25^{*}$ & 0 \\
P22 & 0 & 0 & $0^{*}$ & 0 & $98,75^{*}$ & 1,25 \\
P23 & 0 & 0 & $96,25^{*}$ & 0 & $3,75^{*}$ & 0 \\
P24 & 0 & 1,25 & $0^{*}$ & 1,25 & $97,5^{*}$ & 0 \\
P25 & 0 & 0 & $0^{*}$ & 1,25 & $98,75^{*}$ & 0 \\
P26 & 0 & 0 & $81,25^{*}$ & 0 & $18,75^{*}$ & 0 \\
\hline
\end{tabular}

Nota. C = Reposta à dimensão cor; $E$ = Resposta à espessura; $F=$ Resposta à forma. ${ }^{*}$ Indica sequência correta (reforçada). ${ }^{*}$ Indica sequência incorreta especificada pela instrução discrepante.

A Figura 1 mostra a frequência acumulada de sequências de respostas emitidas por cada participante (P) dos Grupos 1 (P11 a P16) e 2 (P21 a P26), durante as Fases 2 e 3. Pode-se observar que, na Fase 2 do Grupo 1, quando a regra correspondente foi apresentada e o seguimento de regra produzia apenas o reforço social, todos os seis participantes (P11, P12, P13, $\mathrm{P} 14, \mathrm{P} 15$ e $\mathrm{P} 16)$ seguiram a regra durante as quatro sessões desta fase. Isto é, todos emitiram a sequência EFC, especificada por esta regra, em mais de $97 \%$ das tentativas dessa fase, como pode ser observado na Tabela 1.

Na Figura 1 e na Tabela 1, também se pode observar que, na Fase 3 do Grupo 1, quando a regra discrepante foi apresentada, quatro participantes (P12, P13,
P14 e P15) seguiram e dois (P11 e P16) deixaram de seguir a regra discrepante. O Participante P13 seguiu a regra discrepante durante toda a Fase 3, ou seja, emitiu a sequência FCE (especificada pela regra discrepante) em $100 \%$ das tentativas da fase. Já os Participantes P12, P14 e P15, inicialmente seguiram a regra discrepante até as Tentativas 27,11 e 54 , respectivamente. A partir daí, continuaram seguindo a regra discrepante, mas às vezes emitiam a sequência EFC (especificada pela regra correspondente na Fase 2), que produzia o reforço "Muito bem". Deste modo, os Participantes P12, P14 e P15 seguiram a regra discrepante durante a maior parte das tentativas da Fase 3, isto é, emitiram a sequência (FCE) especificada pela regra discrepante em mais de 80,70 e $90 \%$ das tentativas da Fase 3, respectivamente. O Participante P11 

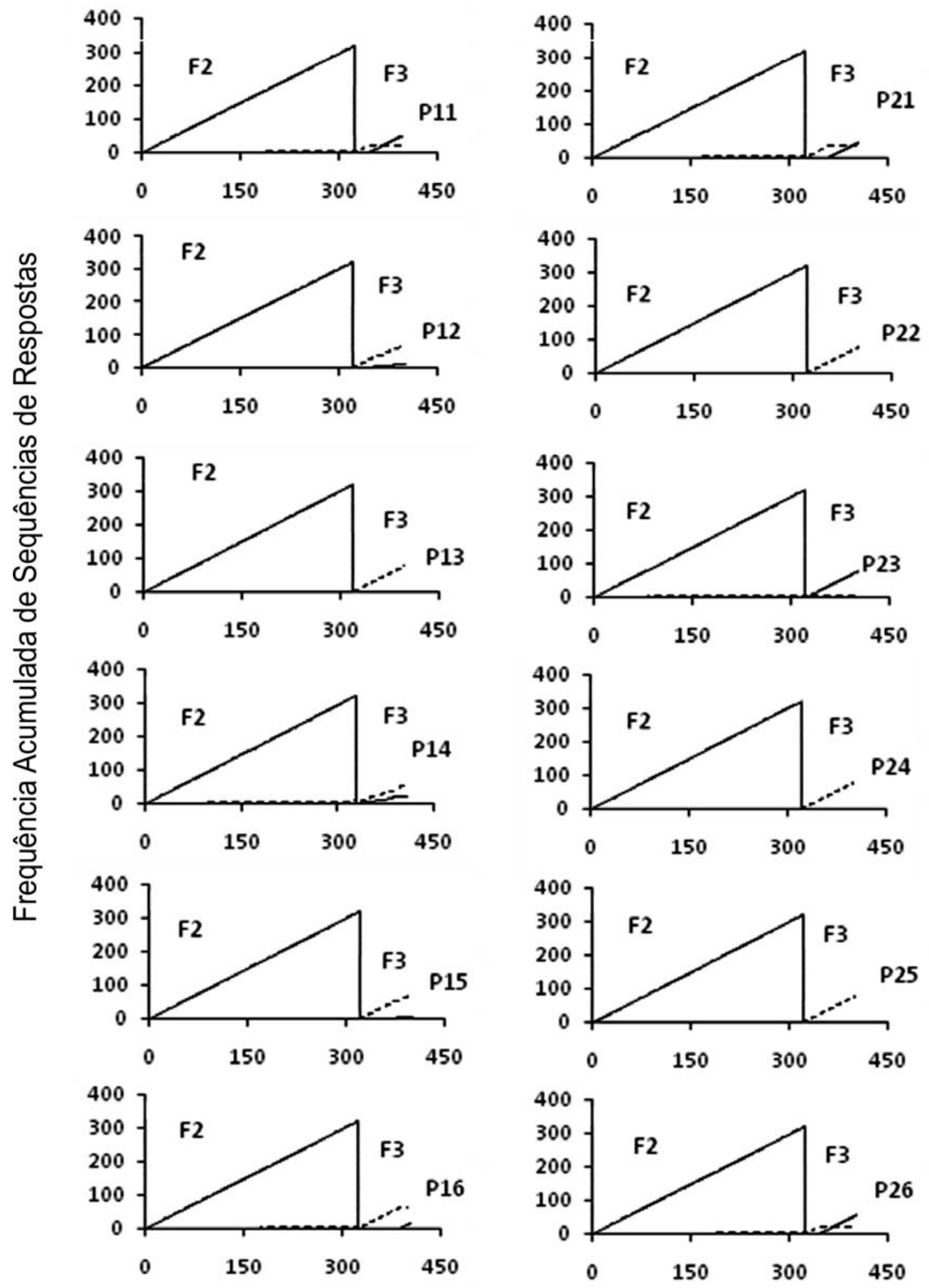

Tentativas

Figura 1. Frequência acumulada de sequências de respostas emitidas por cada participante (P), durante as Fases (F) 2 e 3 dos Grupos 1 (P11 a P16) e 2 (P21 a P26). Linha cheia indica sequência correta e linha tracejada indica sequência incorreta. Quebra na curva acumulada indica mudança de fase. 
seguiu a regra discrepante até a Tentativa 22. A partir daí, abandonou o seguimento desta regra e voltou a emitir a sequência correta EFC (previamente especificada pela regra correspondente) até o final da fase. Deste modo, P11 emitiu a sequência correta em mais de $70 \%$ das tentativas da fase. O Participante P16 emitiu a sequência FCE, especificada pela regra discrepante, em apenas quatro tentativas. No entanto, após deixar de seguir a regra discrepante, este participante passou a emitir outra sequência (CFE) incorreta. A partir da Tentativa 63, entretanto, P16 voltou a emitir a sequência correta EFC, previamente descrita pela regra correspondente, até o final da fase. Deste modo, P16 chegou a emitir a sequência correta em mais de $21 \%$ das tentativas da fase.

Pode-se observar na Figura 1 e na Tabela 2 que, na Fase 2 do Grupo 2, quando a regra correspondente foi apresentada e o seguimento de regra produzia reforço social mais ponto trocável por dinheiro, todos os seis participantes (P21, P22, P23, P24, P25 e P26) seguiram a regra correspondente durante as quatro sessões desta fase. Isto é, todos emitiram a sequência EFC, especificada por esta regra, em mais de $99 \%$ das tentativas dessa fase.

Pode-se observar na Fase 3 do Grupo 2, quando a regra discrepante foi apresentada, que três participantes (P22, P24 e P25) seguiram e três (P21, P23 e P26) deixaram de seguir a regra discrepante. Os Participantes P22, P24 e P25 seguiram a regra discrepante durante toda a Fase 3. Portanto, emitiram a sequência FCE em 100\% das tentativas. Já os Participantes P21, P23 e P26 seguiram a regra discrepante até as Tentativas 35,3 e 15, respectivamente. A partir daí, passaram a emitir a sequência correta EFC (especificada pela regra correspondente na Fase 2) até o final desta fase. Assim, P21, P23 e P26 abandonaram o seguimento da regra discrepante e passaram a emitir a sequência especificada pela regra correspondente em mais de 56 , 96 e $80 \%$ das tentativas da fase, respectivamente.

\section{DISCUSSÃO}

Os resultados do presente estudo, juntos com os resultados obtidos no estudo de Albuquerque e cols. (2008), sugerem que uma história de reforço social para o seguimento de regra correspondente às contingências pode contribuir para manter o seguimento subsequente de regras discrepantes das contingências. Ou seja, quando, antes de serem expostos à regra discrepante, os participantes foram expostos a uma história ao longo da qual o seguimento de regra produzia ponto trocável por dinheiro, nenhum (correspondente a $0 \%$ ) dos quatro participantes seguiu a regra discrepante (caso do estudo de Albuquerque e colaboradores). Mas quando, antes de serem expostos à regra discrepante, os participantes foram expostos a uma história ao longo da qual o seguimento de regra produzia reforço social ou reforço social mais ponto trocável por dinheiro, 7 de 12 (correspondente a 58\%) participantes seguiram a regra discrepante (caso do presente estudo).

Os resultados dos estudos de Albuquerque e cols. (2008) e Albuquerque e cols. (2006) mostraram que os efeitos de uma história de reforço contínuo para o seguimento de regra correspondente sobre o seguimento subsequente de regras discrepantes das contingências dependem, em parte, da extensão dessa história. Isto quando a extensão dessa história é medida pelo número de reforços fornecidos para a emissão do comportamento estabelecido pela regra correspondente. Por essa proposição, o controle por uma história de reforço contínuo de um comportamento estabelecido por regra correspondente tem maior probabilidade de impedir que o seguimento subsequente de uma regra discrepante das contingências seja mantido, quando essa história é longa (história encerrada após o fornecimento de 320 reforços) do que quando ela é curta (história encerrada após o fornecimento de 80 reforços).

Já os resultados do presente estudo e do estudo de Albuquerque e cols. (2008) sugerem, em adição, que os efeitos de uma história prolongada de reforço contínuo para o seguimento da regra correspondente sobre o seguimento subsequente de regras discrepantes das contingências também dependem, em parte, do tipo de consequência produzida pelo comportamento estabelecido pela regra correspondente. Por essa proposição, o controle por uma história prolongada de reforço contínuo de um comportamento estabelecido por regra correspondente tem maior probabilidade de manter o seguimento subsequente de uma regra discrepante das contingências, quando as consequências produzidas pelo comportamento estabelecido pela regra correspondente envolvem a aprovação social do falante que apresentou a regra (como quando esse comportamento produz a expressão "Muito bem") do que quando as consequências produzidas por esse comportamento não envolvem tal aprovação social (como quando esse comportamento produz apenas pontos trocáveis por dinheiro).

Pode-se dizer, portanto, que nem toda história de reforço para o seguimento de regra contribui para a manutenção do seguimento subsequente de outras regras e que manter o seguimento subsequente de 
outras regras não é o único efeito que uma história de reforço para o seguimento de regra pode exercer. Os resultados em análise apoiam a sugestão de que uma história de reforço para o seguimento de regras pode contribuir tanto para manter quanto para substituir $\mathrm{o}$ controle subsequente por regras discrepantes das contingências (Albuquerque, 1998; Albuquerque \& cols., 2003; Oliveira \& Albuquerque, 2007). Mas, além disso, como indicado no parágrafo anterior, eles também contribuem para identificar algumas das condições sob as quais um ou outro desses dois efeitos teria maior ou menor probabilidade de ser exercido.

Os resultados em análise também apoiam a sugestão de que regras são seguidas devido a uma história de reforço social para o seguimento de regras (Albuquerque \& cols., 2004; Baron \& Galizio, 1983; Catania \& cols., 1990; Catania, Shimoff \& Matthews, 1989; Cerutti, 1989; Chase \& Danforth, 1991; Hayes \& cols., 1986; Joyce \& Chase, 1990; Malott, 1989; Pinto, Paracampo \& Albuquerque, 2006; Otto \& cols., 1999; Skinner, 1969; Torgrud \& Holborn, 1990; Wulfert, Greenway, Farkas, Hayes, \& Douguer, 1994; Zettle \& Hayes, 1982). Isto não implica em dizer que o seguimento de regras seria mantido exclusivamente por uma história de reforço social para o seguimento de regras. Há outras possibilidades (ver Albuquerque, 2005) que deveriam ser investigas experimentalmente.

Por exemplo, de acordo com Albuquerque (2005), o seguimento de regras pode ser determinado, não apenas por uma história de reforço para o seguir e de punição para o não seguir regras, mas também por uma história de controle por regras que relatam justificativas para ocorrência do seguimento de regras. Isto é, por uma história de controle por regras que relatam consequências remotas ou atrasadas, tais como promessas de reforço para o seguir e ameaças de punição para o não seguir regras. Por essa visão, então, uma pessoa também pode seguir regras, por exemplo, porque no passado lhe foram feitas ameaças de que o não seguir regras seria punido, ou porque no passado the foram feitas promessas de que o seguir regras seria reforçado, ou porque no passado fez acordos que especificavam que o seguir regras seria reforçado e o não seguir regras seria punido.

Para Albuquerque (2005), o relato de consequências atrasadas por uma regra faz parte da regra e, portanto, pode exercer controle sobre a ocorrência do comportamento especificado pela regra como um elemento verbal participante da regra, isto é, como estímulo antecedente verbal e não como uma consequência do comportamento. Já o evento futuro relatado pela regra para ela ser seguida e que poderia ocorrer caso a regra fosse seguida, não pode exercer controle sobre o comportamento sob controle antecedente da regra, porque o comportamento não pode ficar sob controle de um evento que ainda não ocorreu. Assim, quando uma regra relata consequências atrasadas, a ocorrência do comportamento especificado pela regra não ficaria sob controle do evento futuro relatado pela regra, mas sim de um evento passado, isto é, da própria regra. O comportamento ficaria sob controle da regra, em parte, devido às histórias de seguimento de regras do ouvinte. Se isto for admitido, deveria ser esperado que a probabilidade do seguimento de regras vir a ocorrer no futuro pudesse depender, em parte, do tipo de consequência relatada na regra, isto é, pudesse depender, em parte, das propriedades formais da própria regra. Uma maneira de testar essas suposições seria manipular diferentes tipos de regras, enquanto as contingências programadas fossem mantidas inalteradas. Pesquisas futuras também poderiam avaliar se histórias de seguimento de regras, apresentadas na forma de ameaças ou de promessas, também poderiam contribuir, tal como histórias de reforço social, para aumentar a probabilidade do seguimento subsequente de regras discrepantes das contingências ser mantido. Em todos os casos, para testar essas possibilidades, seria indicado utilizar o procedimento usado no presente estudo e no estudo de Albuquerque e cols. (2008), porque isso tornaria os dados dos três estudos comparáveis.

\section{REFERÊNCIAS}

Albuquerque, L. C. (1998). Efeitos de histórias experimentais sobre o seguimento subsequentes de regras. Tese de doutorado não-publicada, Universidade de São Paulo.

Albuquerque, L. C. (2005). Regras como instrumento de análise do comportamento. Em L. C. Albuquerque (Org.), Estudos do comportamento (pp. 143-176). Belém: EDUFPA.

Albuquerque, L. C., de Souza, D. G., Matos, M. A., \& Paracampo, C. C. P. (2003). Análise dos efeitos de histórias experimentais sobre o seguimento subsequente de regras. Acta Comportamentalia, 11, 87-126.

Albuquerque, L. C., Matos, M. A., de Souza, D. G., \& Paracampo, C. C. P. (2004). Investigação do controle por regras e do controle por histórias de reforço sobre o comportamento humano. Psicologia: Reflexão e Crítica, 17, 395-412.

Albuquerque, L. C., Reis, A. A., \& Paracampo, C. C. P. (2006). Efeitos de uma história de reforço contínuo sobre o seguimento de regra. Acta Comportamentalia, 14, 47-75.

Albuquerque, L. C., Reis, A. A., \& Paracampo, C. C. P. (2008). Efeitos de histórias de reforço, curtas e prolongadas, sobre o seguimento de regras. Acta Comportamentalia, 16, 305-332.

Baron, A., \& Galizio, M. (1983). Instructional control of human operant behavior. The Psychological Record, 33, 495-520. 
Catania, A. C., Matthews, A., \& Shimoff, E. (1990). Properties of rule-governed behaviour and their implications. Em D. E. Blackman \& H. Lejeune (Orgs.), Behaviour analysis in theory and practice: Contributions and controversies (pp. 215-230). Brighton: Lawrence Erlbaum.

Catania, A. C., Shimoff, E., \& Matthews, A. (1989). An experimental analysis of rule-governed behavior. Em S. C. Hayes (Org.), Rule-governed behavior: Cognition, contingencies, and instructional control (pp. 119-150). New York: Plenum.

Cerutti, D. T. (1989). Discrimination theory of rule-governed behavior. Journal of the Experimental Analysis of Behavior, $51,259-276$.

Chase, P. N., \& Danforth, J. S. (1991). The role of rules in conceptlearning. Em L. J. Hayes \& P. N. Chase (Orgs.), Dialogues on verbal behavior (pp. 205-225). Hillsdale, NJ: Erlbaum.

DeGrandpre, R. J., \& Buskist, W. F. (1991). Effects of accuracy of instructions on human behavior: Correspondence with reinforcement contingencies matters. The Psychological Record, 41, 371-384.

Dixon, M. R., \& Hayes, L. J. (1998). Effects of differing instructional histories on the resurgence of rule-following. The Psychological Record, 48, 275-292.

Hayes, S. C., Brownstein, A. J., Zettle, R. D., Rosenfarb, I., \& Korn, Z. (1986). Rule governed behavior and sensitivity to changing consequences of responding. Journal of the Experimental Analysis of Behavior, 45, 237-256.

Joyce, J. H., \& Chase, P. N. (1990). Effects of response variability on the sensivity of rule-governed behavior. Journal of the Experimental Analysis of Behavior, 54, 251-262.

LeFrancois, J. R., Chase, P. N., \& Joyce, J. (1988). The effects of variety of instructions on human fixed-interval performance. Journal of the Experimental Analysis of Behavior, 49, 383-393.

Malott, R. M. (1989). Achievement of evasive goals. Em S. C. Hayes (Org.), Rule governed behavior: Cognition, contingencies, and instructional control. (pp. 153-190). New York: Plenum.

Martinez, H., \& Tomayo, R. (2005). Interactions of contingencies, instructional accuracy, and instructional history in conditional discrimination. The Psychological Record, 55, 633-646.
Michael, R. L., \& Bernstein, D. J. (1991). Transient effects of acquisition history on generalization in a matching-to-sample task. Journal of the Experimental Analysis of Behavior, 56, 155-166.

Okoughi, H. (1999). Instructions as discriminative stimuli. Journal of the Experimental Analysis of Behavior, 72, 205-214.

Oliveira, V. L., \& Albuquerque, L. C. (2007). Efeitos de histórias experimentais e de esquemas de reforço sobre o seguir regras. Psicologia: Teoria e Pesquisa, 23, 217-228.

Otto, T. L., Torgrud, L. J., \& Holborn, S. W. (1999). An operant blocking interpretation of instructed insensitivity to schedule contingencies. The Psychological Record, 49, 663-684.

Pinto, A. R., Paracampo, C. C. P., \& Albuquerque, L. C. (2006). Análise do controle por regras em participantes classificados de flexíveis e de inflexíveis. Acta Comportamentalia, 14, 171194.

Santos, J. G. W., Paracampo, C. C. P., \& Albuquerque, L. C. (2004). Análise dos efeitos de histórias de variação comportamental sobre o seguimento de regras. Psicologia: Reflexão e Crítica, 17, 413-425.

Skinner, B. F. (1969). Contingencies of reinforcement: A theoretical analysis. New York: Appleton-Century-Crofts.

Skinner, B. F. (1974). About behaviorism. New York: Alfred A. Knopf.

Torgrud, L. J., \& Holborn, S. W. (1990). The effects of verbal performance descriptions on nonverbal operant responding. Journal of the Experimental Analysis of Behavior, 54, 273-291.

Wulfert, E., Greenway, D. E., Farkas, P., Hayes, E. C., \& Douguer, M. J. (1994). Correlation between self-reported rigidity and rule-governed insensitivity to operant contingencies. Journal of Applied Behavior Analysis, 27, 659671.

Zettle, R. D., \& Hayes, S. C. (1982). Rule-governed behavior: A potential theoretical framework for cognitive-behavior therapy. Em P. C. Kendall (Org.), Advances in cognitive-behavioral research and therapy (pp. 73-118). New York: Academic Press.

Recebido: 19/06/2009

Última revisão: 25/10/2009

Aceite final: 10/11/2009

\section{Sobre os autores:}

Luiz Carlos de Albuquerque: Professor Associado da Universidade Federal do Pará e do Programa de Pós-Graduação em Teoria e Pesquisa do Comportamento da Mesma Instituição. Bolsista de produtividade em pesquisa do CNPq.

Gilsany Leão Matsuo: Estudante do curso de Psicologia da UFPa e bolsista de iniciação científica do CNPq.

Carla Cristina Paiva Paracampo: Professora Associada da Universidade Federal do Pará e do Programa de Pós-Graduação em Teoria e Pesquisa do Comportamento da mesma instituição. Bolsista de produtividade em pesquisa do CNPq.

Endereço para correspondência: 1calbu@ufpa.br. 